\title{
The Architecture of Reward Value Coding in the Human Orbitofrontal Cortex
}

\author{
Guillaume Sescousse, ${ }^{1,2}$ Jérôme Redouté, ${ }^{1,2,3}$ and Jean-Claude Dreher ${ }^{1,2}$ \\ ${ }^{1}$ Center for Cognitive Neuroscience, Reward and Decision-Making Group, Centre National de la Recherche Scientifique, Unité Mixte de Recherche 5229, \\ 69675 Bron, France, ${ }^{2}$ Université Lyon 1, 69003 Lyon, France, and ${ }^{3}$ CERMEP-Imagerie du Vivant, 69003 Lyon, France
}

To ensure their survival, animals exhibit a number of reward-directed behaviors, such as foraging for food or searching for mates. This suggests that a core set of brain regions may be shared by many species to process different types of rewards. Conversely, many new brain areas have emerged over the course of evolution, suggesting potential specialization of specific brain regions in the processing of more recent rewards such as money. Here, using functional magnetic resonance imaging in humans, we identified the common and distinct brain systems processing the value of erotic stimuli and monetary gains. First, we provide evidence that a set of neural structures, including the ventral striatum, anterior insula, anterior cingulate cortex, and midbrain, encodes the subjective value of rewards regardless of their type, consistent with a general hedonic representation. More importantly, our results reveal reward-specific representations in the orbitofrontal cortex $(\mathrm{OFC})$ : whereas the anterior lateral $\mathrm{OFC}$, a phylogenetically recent structure, processes monetary gains, the posterior lateral $\mathrm{OFC}$, phylogenetically and ontogenetically older, processes more basic erotic stimuli. This dissociation between OFC representations of primary and secondary rewards parallels current views on lateral prefrontal cortex organization in cognitive control, suggesting an increasing trend in complexity along a postero-anterior axis according to more abstract representations. Together, our results support a modular view of reward value coding in the brain and propose that a unifying principle of postero-anterior organization can be applied to the OFC.

\section{Introduction}

A basic concern about the functional organization of the prefrontal cortex is to delineate the functional divisions of the orbitofrontal cortex (OFC). A number of lesion, electrophysiological, and neuroimaging studies indicate a general role for the OFC in encoding the value assigned to different goods in both human (O’Doherty et al., 2003a; Plassmann et al., 2007; Chib et al., 2009; FitzGerald et al., 2009) and nonhuman primates (Tremblay and Schultz, 1999; Padoa-Schioppa and Assad, 2008). An important remaining issue, which is key to our understanding of the functional organization of the OFC, is to determine whether distinct parts of the OFC encode rewards of different nature. The anterior and posterior parts of the OFC are considered to belong to two distinct cytoarchitectonic trends. The anterior part of the OFC, especially well developed in humans and characterized by a granular cell layer, is thought to be phylogenetically and ontogenetically more recent than the posterior and medial parts, which consist of agranular and dysgranular cortices (Ongür and Price, 2000; Wise, 2008). Although never tested empirically, one fundamental hypothesis, based on this increasing trend in complexity

Received July 7, 2010; revised July 19, 2010; accepted Aug. 9, 2010.

This work was funded by a Marie Curie International Reintegration Grant (J.-C.D.) and a Fyssen Foundation Grant (J.-C.D.). G.S. was funded by a scholarship from the French Ministry of Research and the Medical Research Foundation. We thank the staff of CERMEP-Imagerie du Vivant for helpful assistance with data collection.

Correspondence should be addressed to either Guillaume Sescousse or Jean-Claude Dreher, Centre de Neuroscience Cognitive, CNRS UMR 5229, 67 Boulevard Pinel, 69675 Bron Cedex, France, E-mail: gsescousse@isc.cnrs.fr or dreher@isc.cnrs.fr.

DOI:10.1523/JNEUROSCI.3501-10.2010

Copyright $\odot 2010$ the authors $\quad$ 0270-6474/10/3013095-09\$15.00/0 along a postero-anterior axis, is that the anterior part of the OFC would process secondary rewards, whereas the posterior part would process primary rewards (Kringelbach and Rolls, 2004).

Here, we directly tested this hypothesis with functional magnetic resonance imaging (fMRI) by comparing the brain responses to two experienced rewards: money and erotic pictures. These two rewards present significant evolutionary differences likely to be reflected at the cerebral level: whereas money is a secondary reward that appeared recently in human history and whose abstract value needs to be learned by association with primary reinforcers, erotic stimuli can be considered as primary rewards because they have an innate value and satisfy biological needs. We therefore hypothesized that monetary gains would recruit anterior $\mathrm{OFC}$ regions and erotic pictures would engage more posterior OFC regions. Despite their critical sociobiological importance, erotic stimuli have never been studied as reinforcers but rather as arousing stimuli in passive viewing paradigms focusing on sexual function (Redouté et al., 2000; Ponseti et al., 2006). However, erotic stimuli are clearly rewarding (Hamann et al., 2004), probably because sexual attractiveness, which may have evolved to enhance reproductive fitness, is an important cue for mate choice (Thornhill and Gangestad, 1999; Rhodes, 2006).

In addition to specialized OFC regions processing different types of rewards, we hypothesized the existence of common brain structures supporting general hedonic representations independent of reward type. To evaluate and compare the relative value of different rewards on a unique scale, it has been proposed that the brain may use a "common neural currency," likely to be implemented in integrative reward regions such as the ventral striatum 
and ventromedial prefrontal cortex (vmPFC) (Montague and Berns, 2002; Sugrue et al., 2005; Rangel et al., 2008; Dreher, 2009). Consistent with this claim, a number of studies have shown that goal/decision values, reflecting the anticipated rewarding properties of a stimulus at the time of choice, were encoded in these regions regardless of reward type (McClure et al., 2007; Hare et al., 2008; Chib et al., 2009; Peters and Buchel, 2010). However, very few studies have investigated whether shared cerebral substrates are engaged when actually experiencing different types of rewards, i.e., during the computation of outcome/ hedonic values (Izuma et al., 2008; Smith et al., 2010). Such a common representation of outcome value may be particularly useful to support the trading ability demonstrated by primates. For example, humans are willing to sacrifice money to view attractive faces (Hayden et al., 2007), and similarly male monkeys exchange meat for sex or sacrifice fluid for the opportunity to view female perinea (Deaner et al., 2005; Gomes and Boesch, 2009).

Building on these considerations, we designed a reward paradigm aiming to (1) determine whether the OFC is functionally divided depending on reward type and (2) identify common brain structures processing the experienced value of both monetary and erotic rewards. To further characterize the role of these brain regions in reward processing, we also manipulated reward intensity and probability and collected hedonic ratings after reward outcomes inside the scanner. Because erotic pictures are not quantifiable like money, it is unclear whether changes in the intensity of erotic pictures would affect the same brain regions as those responding to changes in monetary amounts. Likewise, it is unknown whether the concept of prediction error, reflecting the discrepancy between expected and actual rewards and primarily used with quantifiable rewards (such as money and juice), can be extended to erotic stimuli.

\section{Materials and Methods}

\section{Participants}

Eighteen right-handed volunteers (mean \pm SD age, $24 \pm 3.3$ years) with no history of neurological or psychiatric disorders participated in this study. All of them were heterosexual males, because men are generally more responsive to visual sexual stimuli than women (Hamann et al., 2004) and to avoid the potential influence of the menstrual cycle known to have an effect on reward processing in women (Caldú and Dreher, 2007; Dreher et al., 2007). All subjects gave written informed consent to be part of the experiment, which was approved by the local ethics committee (Centre Léon Bérard, Lyon, France).

Motivation, which was a crucial element of our study, was closely controlled. First, sexual arousability was assessed at the time of screening through specific questionnaires, namely the Brief Sexual Function questionnaire (Reynolds et al., 1988) and the Sexual Arousability Inventory (Hoon and Chambless, 1998). Of an initial pool of 22 subjects, two of them were excluded because they scored too low on the Sexual Arousability Inventory (mean score for all subjects, $91.1 \pm 14.6$; scores of excluded subjects, 54 and 64). To further ensure that all participants would be in a similar state of motivation to see erotic stimuli, we asked them to avoid any sexual contact during a period of $24 \mathrm{~h}$ before the scanning session. Second, we sought to enhance the motivation for money by telling the subjects that the financial compensation for their participation would be calculated based on their winnings during the task. We also excluded two subjects presenting symptoms of depression as assessed by the 13-item version of the Beck Depression Inventory (Beck and Beck, 1972) (mean score for all subjects, $1.9 \pm 2.7$; scores of excluded subjects, 6 and 10).

Task

Our protocol was inspired from the typical design of incentive delay tasks (Knutson et al., 2005; Abler et al., 2006) but included several modifica- tions related to our questions. Experimental trials were divided into two phases: reward anticipation and outcome. During reward anticipation, a cue was presented, followed by a delay period and a discrimination task (Fig. 1). The cue carried three types of information regarding the upcoming reward: the red portion of a pie chart in the background indicated its probability $(25,50$, or $75 \%)$, and the pictogram in the foreground indicated its type (monetary or erotic) and intensity (high or low, depending on the size of the pictogram). This led to a total of 12 different cues plus a control condition associated with no chance of winning (supplemental Fig. 1, available at www.jneurosci.org as supplemental material). After a variable delay period (question mark representing a pseudorandom draw depending on probability), subjects were asked to perform a discrimination task, in which they had to respond correctly to a target within a maximum time of $1 \mathrm{~s}$. The shape of the target was drawn at random on each trial and could be either a triangle (left button press) or a square (right button press). Success on this discrimination task (indicated by a magnified target) allowed the subjects to view the outcome of the pseudorandom draw, whereas erroneous or slow response (indicated by no change in target size) led to no reward. In rewarded trials, the reward was either an erotic image (with high or low erotic content) or the picture of a safe mentioning the amount of money won (high or low amount). After each reward outcome, subjects were asked to provide a hedonic rating by moving a cursor along a 1-to-9 continuous scale ( 1 for very little pleased; 9 for very highly pleased). In non-rewarded and control trials, the subjects were presented with "scrambled" pictures. A fixation cross was finally used as an intertrial interval of variable length.

\section{Stimuli}

Two categories (high and low intensity) of erotic pictures and monetary gains were used. Nudity being the main criteria driving the reward value of erotic stimuli, we separated them into a "low intensity" group displaying women in underwear or bathing suits and a "high intensity" group displaying naked women in an inviting posture. Each erotic picture was presented only once during the course of the task to avoid habituation. A similar element of surprise was introduced for the monetary rewards by randomly varying the amounts at stake: the low amounts were $€ 1, € 2$, or $€ 3$ and the high amounts were $€ 10, € 11$, or $€ 12$. The pictures displayed in non-rewarded and control trials were scrambled versions of the pictures used in rewarded trials and hence contained the same information in terms of chromaticity and luminance.

\section{fMRI data acquisition}

Imaging was conducted on a $1.5 \mathrm{~T}$ Siemens Sonata scanner, using an eight-channel head coil. The scanning session was divided into four runs. Each of them included four repetitions of each cue, with the exception of the control condition, repeated nine times. This yielded a total of 228 trials. Within each run, the order of the different conditions was pseudorandomized and optimized to improve signal deconvolution. The order of the runs was counterbalanced between subjects. Before scanning, all subjects were given oral instructions and familiarized with the cognitive task in a short training session.

Each of the four functional runs consisted of 296 volumes. Twenty-six interleaved slices parallel to the anterior commissure-posterior commissure line were acquired per volume (field of view, $220 \mathrm{~mm}$; matrix, $64 \times$ 64 ; voxel size, $3.4 \times 3.4 \times 4 \mathrm{~mm}$; gap, $0.4 \mathrm{~mm}$ ), using a gradient-echo echoplanar imaging (EPI) T2*-weighted sequence (repetition time, 2500 $\mathrm{ms}$; echo time, $60 \mathrm{~ms}$; flip angle, $90^{\circ}$ ). To improve the local field homogeneity and hence minimize susceptibility artifacts in the orbitofrontal area, a manual shimming was performed within a rectangular region including the OFC and the basal ganglia. A high-resolution T1-weighted structural scan was subsequently acquired in each subject.

\section{fMRI analysis}

Preprocessing. Preprocessing of fMRI data was conducted using SPM2. The first four functional volumes of each run were removed, and the remaining images were corrected for slice-timing artifacts and spatially realigned to the first image of each time series. We then searched for residual artifacts in the time series with the tsdiffana utility (http:// imaging.mrc-cbu.cam.ac.uk/imaging/DataDiagnostics) and modeled them with dummy regressors in our general linear model (two subjects 
A

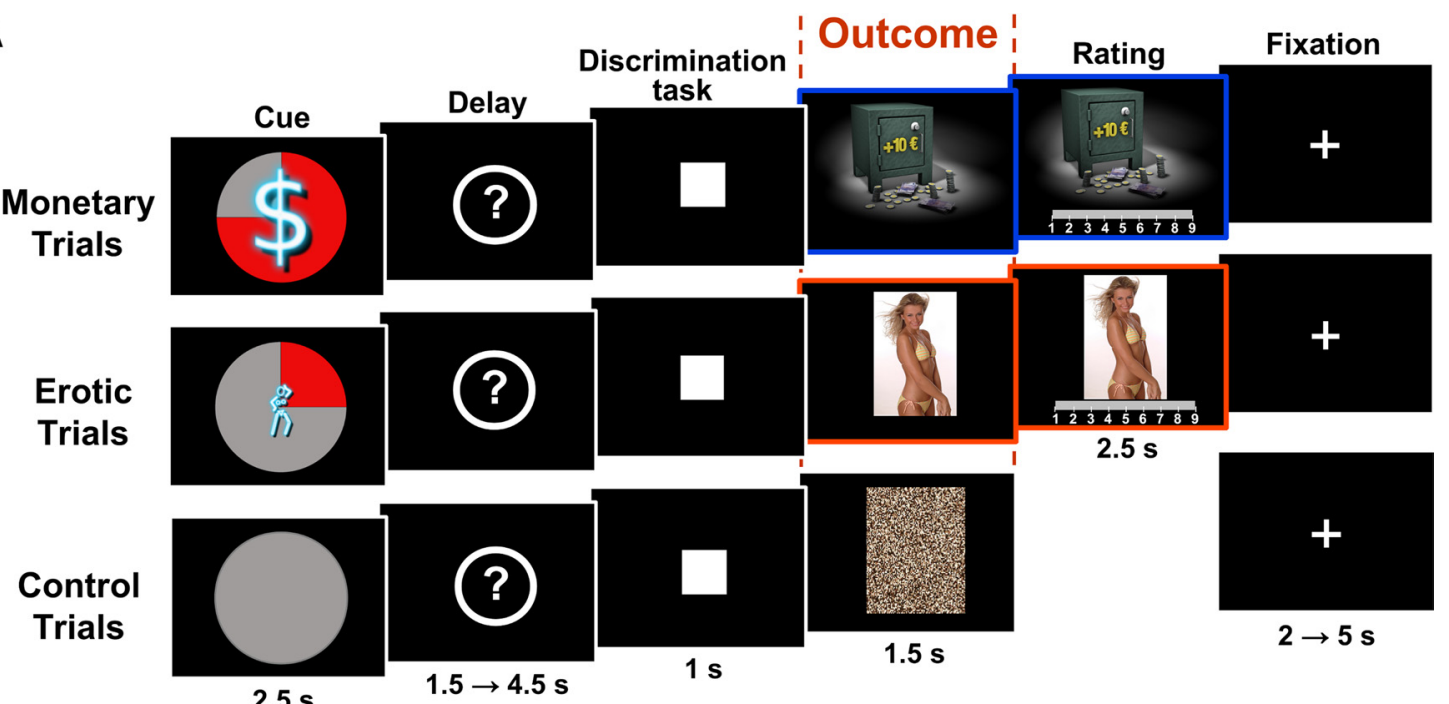

B



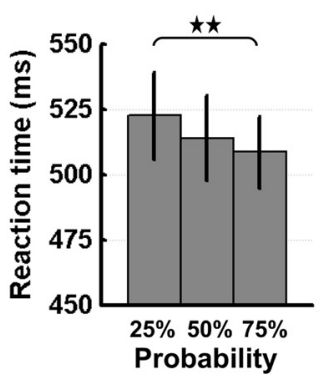

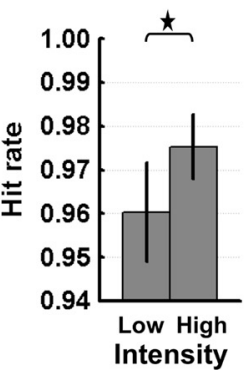

C Monetary reward

Erotic reward

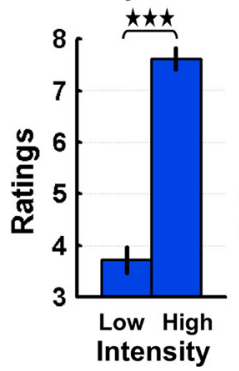

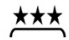

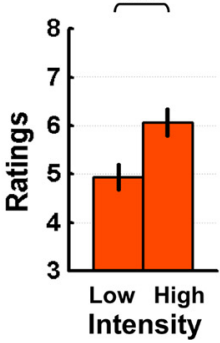

Figure 1. Paradigm and behavior. A, Sequence of events during a typical trial. Subjects first saw a cue informing them about the type, probability, and intensity of an upcoming reward (supplemental Fig. 1, available at www.jneurosci.org as supplemental material). Three cases are represented here: a $75 \%$ chance of receiving a high amount of money (top), a $25 \%$ chance of seeing a low erotic content picture (middle), and a sure chance of getting nothing (control trials; bottom). After a short delay and a target discrimination task, subjects saw the outcome, which was contingent on both the announced probability and their performance on the discrimination task. Reward outcomes consisted either in a monetary amount displayed on a safe (top) or an erotic picture (middle) and were followed by the rating of their subjective value on a continuous scale. Non-rewarded and control trials displayed a scrambled picture at outcome (bottom). $\boldsymbol{B}$, Behavioral results on the discrimination task: mean reaction times according to reward intensity (left) and probability (middle) and mean hit rates according to reward intensity (right). $C$, Mean subjective ratings according to reward intensity, on a 1-to-9 scale. Error bars indicate SEM. ${ }^{*} p<0.05 ;{ }^{* *} p<0.01$; ${ }^{* * *} p<0.001$ by Tukey's HSD tests.

had three artifacts and one subject had one artifact in their time series). The functional images were then normalized to the Montreal Neurological Institute (MNI) stereotaxic space using the EPI template of SPM2 and spatially smoothed with a $10 \mathrm{~mm}$ full-width at half-maximum isotropic Gaussian kernel. Anatomical scans were normalized to the MNI space using the icbm152 template brain and averaged across subjects.

Identification of common and specific brain regions. The event-related statistical analysis was performed according to the general linear model as implemented in SPM2. Anticipation-related responses were modeled as boxcar functions time locked to the onset time of the cue with a duration of $2.5 \mathrm{~s}$. The 2 rewards (monetary/erotic) $\times 2$ intensities (high/ low) were modeled as four separate conditions. For each of them, a first-order parametric regressor modeled reward probability. The control condition was modeled in a separate regressor. Outcome-related responses were modeled as events time locked to the appearance of the reward (or scrambled picture). Four main conditions were defined: "monetary reward" (MR), "erotic reward" (ER), "no-monetary reward" (NoMR), and "no-erotic reward" (NoER). Two orthogonalized covariates linearly modeling the expected probability and the ratings were added (in this order) to the MR and ER regressors. A last regressor modeled the appearance of a scrambled picture in the control condition (C). All regressors were subsequently convolved with the canonical hemodynamic response function and entered in a first-level analysis. A high-pass filter with a cutoff of $128 \mathrm{~s}$ was applied to the time series to remove low-frequency noise and baseline drifts. The resulting images of parameter estimates were then passed in a secondlevel group analysis in which between-subject variability was treated as a random effect.
Although the anticipatory period was explicitly modeled in our analysis, we only report results concerning the outcome phase because our focus was on the coding of experienced reward value. Following the forward inference approach (Henson, 2006) (see Results), brain regions responding specifically to monetary (or erotic) rewards resulted from the contrast $\mathrm{MR}>\mathrm{ER}$ (or ER $>\mathrm{MR}$ ), masked inclusively with $\mathrm{MR}>\mathrm{C}$ (or $\mathrm{ER}>\mathrm{C}$ ) and exclusively with ER $>\mathrm{C}$ (or MR $>\mathrm{C}$ ). The main contrasts $\mathrm{MR}>\mathrm{ER}$ and $\mathrm{ER}>\mathrm{MR}$ and the masks were thresholded independently using a whole-brain correction for multiple comparisons $[p<0.05$ familywise error (FWE) and $p<0.01$ false discovery rate (FDR), respectively], thereby ensuring the absence of any "selection bias" in the analysis (Kriegeskorte et al., 2009). Brain regions activated by both monetary and erotic rewards were identified in two steps. We first performed a conjunction analysis of the contrasts MR $>\mathrm{C}$ and ER $>\mathrm{C}$ based on the minimum statistic (Nichols et al., 2005) $(p<0.05$ FWE whole-brain corrected). Because this conjunction may be sensitive to other types of computations (such as attention or image processing), we then masked it inclusively with the regions responding parametrically with both monetary and erotic hedonic ratings (each mask thresholded at $p<0.05$ FDR whole-brain corrected).

Anatomical localization of functional clusters was performed based on a probabilistic atlas (Hammers et al., 2003).

Prediction error model. Positive prediction errors were defined at each trial $t$ by $P E(t)=V(t)-B(t)$, where $V(t)$ is the outcome value and $B(t)$ is the expected value (Yacubian et al., 2006). Whereas monetary amounts could have been used to assess $V(t)$ for monetary rewards, erotic rewards could not be similarly quantified. Hence, to use an equivalent measure for both rewards, we used the hedonic rating to assess $V(t)$ on each trial. 


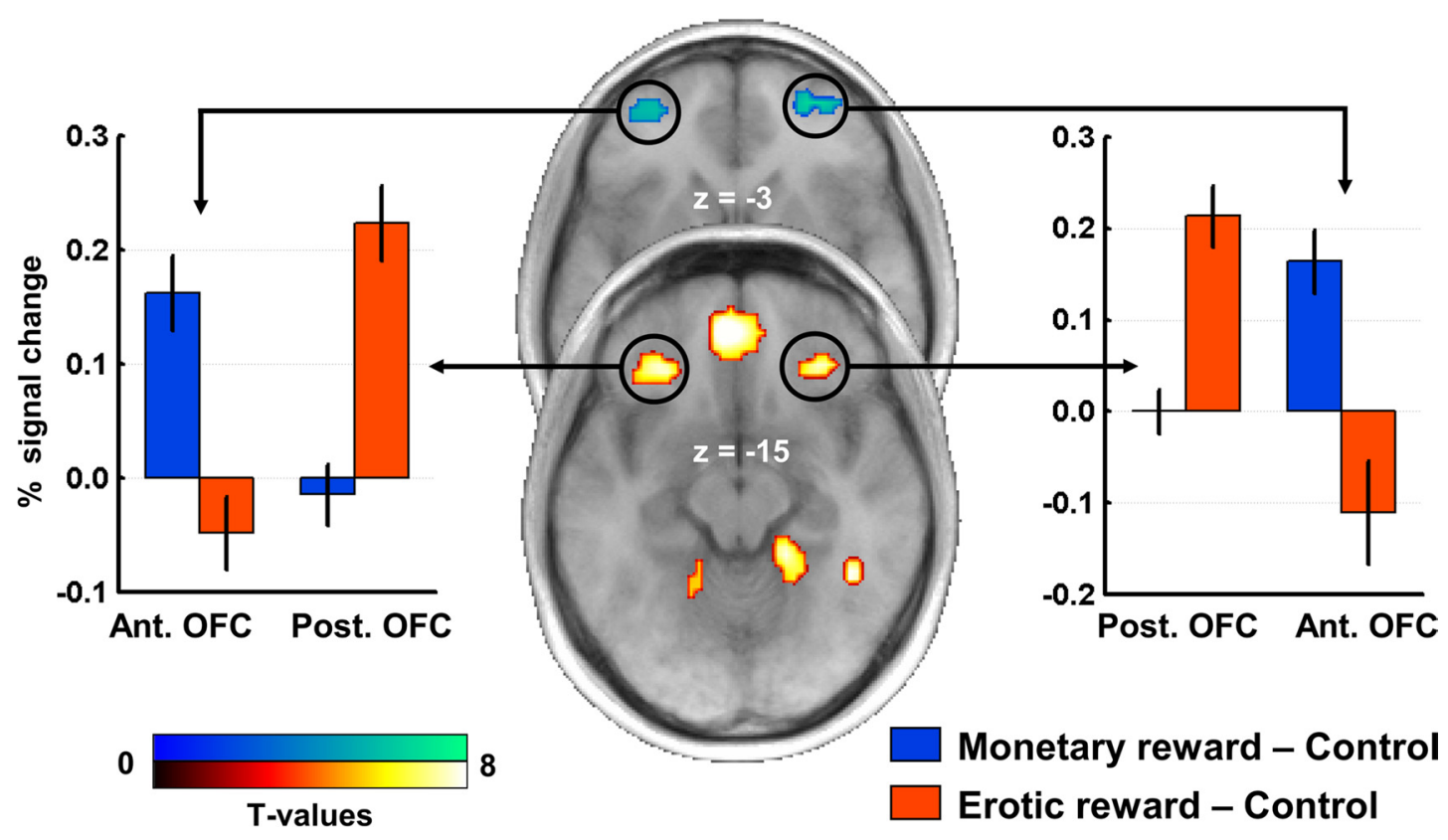

Figure 2. Functional postero-anterior dissociation in the orbitofrontal cortex depending on reward type. Brain regions responding specifically to monetary reward outcomes are displayed in blue- green, and those responding specifically to erotic reward outcomes are displayed in red-yellow. Plots of mean percent signal change, which are not independent of the whole-brain analysis, are shown only to illustrate the double dissociation between monetary/erotic rewards and anterior (Ant.)/posterior (Post.) OFC. Activations are overlaid on an average anatomical scan of all subjects ( $p<0.05$ FWE whole-brain corrected). Error bars indicate SEM.

$B(t)$ was defined as the product of reward probability $P(t)$ by expected intensity $E(t) . P(t)$ was simply the probability given explicitly in the cue $(25,50$, or $75 \%) . E(t)$, presented as either "high" or "low" to the participants, was transformed into a numerical value by using the past ratings: for instance, $E(t)$ for a high monetary reward was estimated as the average of all the ratings given to high monetary rewards since the beginning of the task up to trial $t$.

Prediction error values were entered into two parametric regressors separately modeling monetary and erotic reward prediction errors $\left(\mathrm{PE}_{\mathrm{MR}}\right.$ and $\left.\mathrm{PE}_{\mathrm{ER}}\right)$. Note, however, that prediction error was relatively correlated with outcome value, i.e., with the hedonic ratings (mean $r=$ 0.72 for monetary rewards and mean $r=0.75$ for erotic rewards). This correlation is inherent to the nature of these signals and is a classical shortcoming of fMRI studies on reward processing (Hare et al., 2008). As a consequence, if prediction errors and hedonic ratings were entered in the same general linear model, they would both end up with a rather low explanatory power, because only the orthogonal component of each regressor would be allowed to compete for variance (Hunt, 2008) (such a model was estimated and produced poor results in expected brain regions such as the ventral striatum and the $\mathrm{OFC}$ ). For this reason, we built a separate general linear model, in which reward outcomes were modulated only by prediction errors (instead of probability and ratings). We should emphasize, however, that this procedure makes it difficult to distinguish the contribution of prediction error and outcome value computations at the brain level.

The resulting $T$-maps showing positive correlations between the blood oxygenation level-dependent (BOLD) signal and monetary or erotic prediction errors were subsequently entered in a conjunction analysis (Nichols et al., 2005) thresholded at $p<0.001$ uncorrected for multiple comparisons at the voxel level.

Region of interest analyses. Region of interest (ROI) analyses were conducted with the extension of SPM MarsBaR (http://marsbar.sourceforge. net/) within ROIs defined functionally from the whole-brain analyses. Each ROI was created by taking the intersection of the functional cluster of interest and a 10-mm-radius sphere centered on the highest peak voxel of the cluster (to isolate distinct brain areas pertaining to the same cluster). In keeping with the approach of MarsBaR, percent signal change for a given condition in a given ROI was calculated as the effect size of that

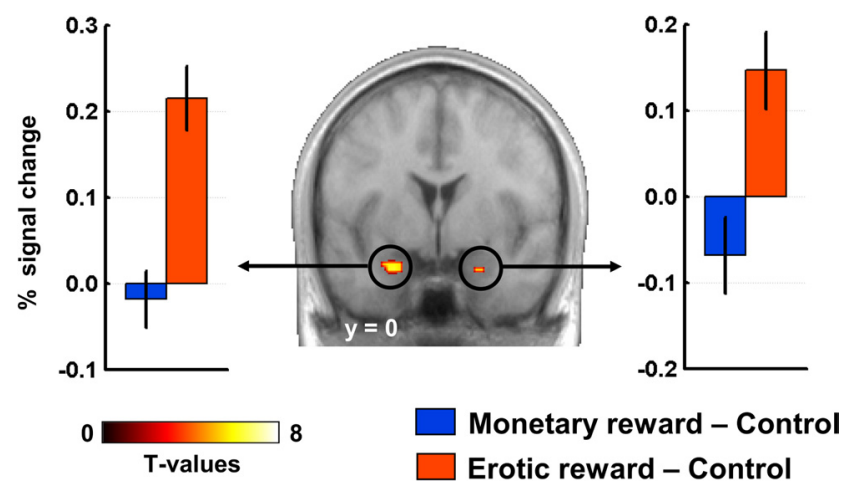

Figure 3. Specific response of amygdala to erotic rewards. Activations are overlaid on an average anatomical scan of all subjects ( $p<0.05 \mathrm{FWE}$ whole-brain corrected). Left and right plots of mean percent signal change, which are not independent of the whole-brain analysis, are shown only to illustrate the specificity of amygdalar response. Error bars indicate SEM.

condition ( $\beta$ value) divided by the mean activity of that ROI and multiplied by 100 .

\section{Results}

\section{Behavior}

Hit rates and reaction times (RTs), obtained at the time of the discrimination task, as well as hedonic ratings obtained at the time of outcome, were analyzed in separate three-way ANOVAs including reward type, probability, and intensity as withinsubject factors. The analysis on hit rates and RT was performed on 17 subjects only, because data were accidentally lost for one subject. The mean hit rate across subjects on the discrimination task was $96 \%$.

There was no significant main effect of reward type on hit rates $(p=0.38)$ and $\mathrm{RT}(p=0.20)$, suggesting that monetary gains and erotic pictures had comparable incentive values. 


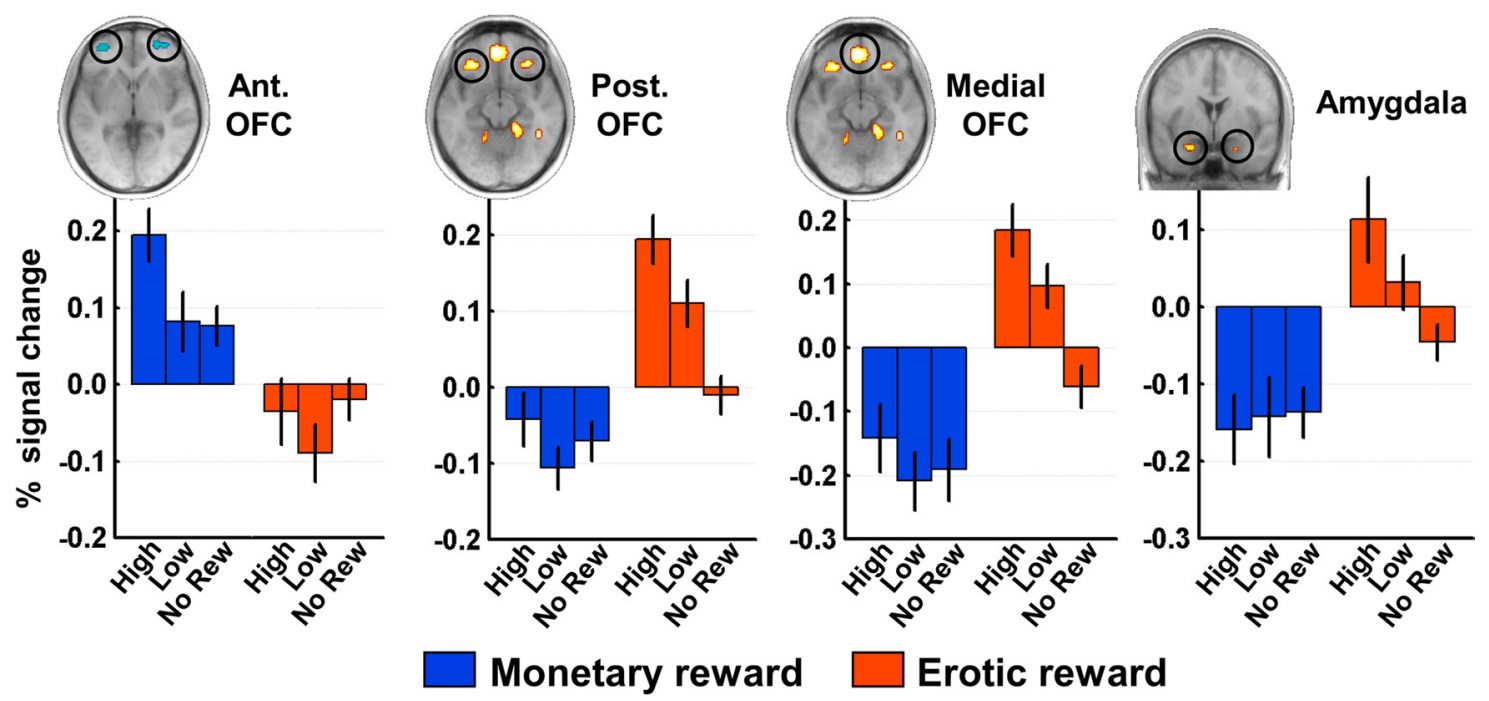

Figure 4. Response pattern of the reward-specific brain regions as a function of reward intensity. Percent signal change is plotted in the circled ROls for monetary and erotic rewards according to the following conditions: high intensity, low intensity, and no reward. In each region, brain activity increases with reward intensity only for the reward for which it is specific. Error bars indicate SEM. The signal is averaged across the right and left hemispheres in each brain region (similar patterns of activity were observed in each hemisphere). Ant., Anterior; Post., posterior.

Subjects were faster $\left(F_{(1,16)}=34.2, p<0.001\right)$ and more accurate $\left(F_{(1,16)}=7.7, p<0.05\right)$ for high intensity incentives and were also faster for more likely rewards $\left(F_{(2,32)}=5.3, p<0.05\right)$ (Fig. $1 B$ ). These results reflect increased motivation for higher reward intensity and more certain rewards. They also confirm that subjects were engaged in the task and effectively encoded the cue information. Importantly, these effects were similar for monetary and erotic rewards, as shown by the absence of significant interaction between intensity and reward type (hit rate, $p=0.67$; RT, $p=0.20$ ) (supplemental Fig. 2, available at www.jneurosci. org as supplemental material) and between probability and reward type (RT, $p=0.11$ ).

No significant effect of reward type was observed on the hedonic ratings $(p=0.40)$, suggesting that monetary and erotic rewards had similar subjective values. Conversely, we found a robust main effect of intensity on the ratings $\left(F_{(1,17)}=150.8, p<\right.$ $0.001)$, which remained significant for each type of reward taken separately [Tukey's honestly significant difference (HSD) tests: monetary rewards, $T_{(17)}=20.4, p<0.001$; erotic rewards, $T_{(17)}=4.4, p<0.001$ ] (Fig. $1 C$ ). This shows that, for both rewards, the two intensity categories chosen a priori (high vs low) were effectively perceived by the subjects. The ratings also showed an interaction between reward type and intensity $\left(F_{(1,17)}=111.5\right.$, $p<0.001)$, simply because the subjects used a smaller portion of the scale to rate erotic pictures. Finally, the ratings were not influenced by reward probability $\left(F_{(2,34)}=1.4, p=0.26\right)$, confirming that they reflected a purely hedonic evaluation.

\section{Neuroimaging data}

Reward-specific brain regions

Brain regions specific for each type of reward were identified based on the forward inference approach proposed by Henson (2006) to demonstrate qualitative differences in brain imaging data. Specifically, money-specific regions were defined as those stemming from the comparison $\mathrm{MR}>\mathrm{ER}$, masked inclusively with $\mathrm{MR}>\mathrm{C}$ and exclusively with $\mathrm{ER}>\mathrm{C}$, and conversely eroticspecific regions were defined as those responding in the comparison $\mathrm{ER}>\mathrm{MR}$, masked inclusively with $\mathrm{ER}>\mathrm{C}$ and exclusively with $\mathrm{MR}>\mathrm{C}$. This procedure ensures that the resulting brain areas meet two criteria: (1) they are more activated by one reward compared with the other ("dissociation" criterion); (2) they respond to either monetary or erotic rewards, but not to both, compared with a common control condition ("association" criterion).

As hypothesized, monetary rewards specifically recruited the anterior lateral OFC (MNI $[x y z][-30,51,0], \mathrm{T}=5.92$; [30, 54, -3 ], $T=6.80)$, spanning the anterior orbital gyrus, the lateral orbital gyrus, and the ventral part of the middle frontal gyrus (Fig. 2). In contrast, erotic rewards elicited activity specifically in the posterior part of the lateral OFC $([-30,33,-15], T=7.56$; [30, $33,-15], \mathrm{T}=7.54)$, straddling the posterior and lateral orbital gyri (Fig. 2). These results demonstrate a double dissociation between monetary/erotic rewards and the anterior/posterior OFC, which is further illustrated in the bar graphs of Figure 2, representing the $\mathrm{MR}>\mathrm{C}$ and $\mathrm{ER}>\mathrm{C}$ differences in percent signal change extracted from these regions. Among eroticspecific areas, a large cluster was also present in the medial OFC ( $[-6,45,-15], T=8.90)$, encompassing the medial orbital gyrus, the straight gyrus, and the most ventral part of the superior frontal gyrus (Fig. 2). Subcortically, the only structure specifically activated by erotic pictures was the bilateral amygdala ([ $-21,-6$, -27], $T=6.94$; [24, 0, -27], $T=5.45$ ) (Fig. 3). Other moneyspecific and erotic-specific foci are reported in supplemental Tables 1 and 2 (available at www.jneurosci.org as supplemental material), respectively.

This segregated representation of reward types in the OFC was not merely attributable to visual or hedonic differences between monetary and erotic outcomes, because an identical dissociation emerged when we repeated the same analysis using no-reward outcomes instead of reward outcomes. That is, when comparing the NoMR and NoER conditions, which only differ with respect to the type of reward being expected (while comparing visually identical scrambled pictures), the anterior OFC specifically responded to no-monetary outcomes $([-30,51,0], T=6.16$; [33, $51,0], T=6.20)$, whereas the posterior OFC specifically responded to no-erotic outcomes ( $[-21,33,-12], T=6.39)$ (supplemental Fig. 3, supplemental Tables 1, 2, available at www. jneurosci.org as supplemental material). This result excludes a mere 
perceptual account of the functional dissociation observed in the OFC and supports the idea that monetary and erotic rewards are encoded in distinct OFC regions.

Moreover, ROI analyses on reward intensity coding brought additional evidence in support of a segregated representation of monetary and erotic outcomes. For both rewards, we extracted the percent signal change for the high reward, low reward, and no-reward conditions (Fig. 4). The results show that, whereas activity in the anterior OFC increased with monetary reward intensity, such a monotonic variation was not present for increasing levels of erotic reward intensity. Conversely, in the posterior OFC, medial OFC, and amygdala, activity was found to increase monotonically with erotic reward intensity but not with monetary reward intensity. Together, these findings indicate that reward-specific brain regions only reflected intensity for the reward type they specifically encoded.

Finally, to further confirm the specificity of these regions with respect to the subjective experience of monetary and erotic outcomes, we performed a whole-brain analysis comparing the coding of their hedonic value. For each reinforcer, reward and no-reward outcomes were pooled together, while the hedonic value was modeled in a parametric regressor with the corresponding continuous rating (from 1 to 9 ) or a 0 , respectively. As expected, the contrast of these parametric regressors between the two rewards revealed the same brain regions as the previous categorical analysis. In particular, BOLD activity in the right anterior lateral OFC was found to scale best with monetary hedonic value, whereas activity in the posterior lateral OFC, medial OFC, and amygdala was found to scale best with erotic hedonic value (supplemental Fig. 4, available at www.jneurosci.org as supplemental material).

\section{Common reward brain regions}

To identify the brain regions commonly activated by monetary and erotic outcomes, we first compared each reward with the control condition and performed a conjunction of these two comparisons (supplemental Fig. 5, available at www.jneurosci.org as supplemental material). Because this analysis remains sensitive to non-reward-related computations such as attentional or image processing effects, it was masked by the brain regions responding parametrically with the subjective value of both monetary and erotic rewards (i.e., hedonic ratings) (Fig. 5). This procedure revealed significant bilateral activations in a set of brain regions classically involved in reward processing: the ventral striatum $([-12,9,-9], T=6.38 ;[9,6$, -9], $T=6.20)$, the midbrain $([-3,-24,-24], T=7.30)$, the anterior cingulate cortex (ACC) $[-6,27,39], T=8.15 ;[9,18$, Ant., Anterior.

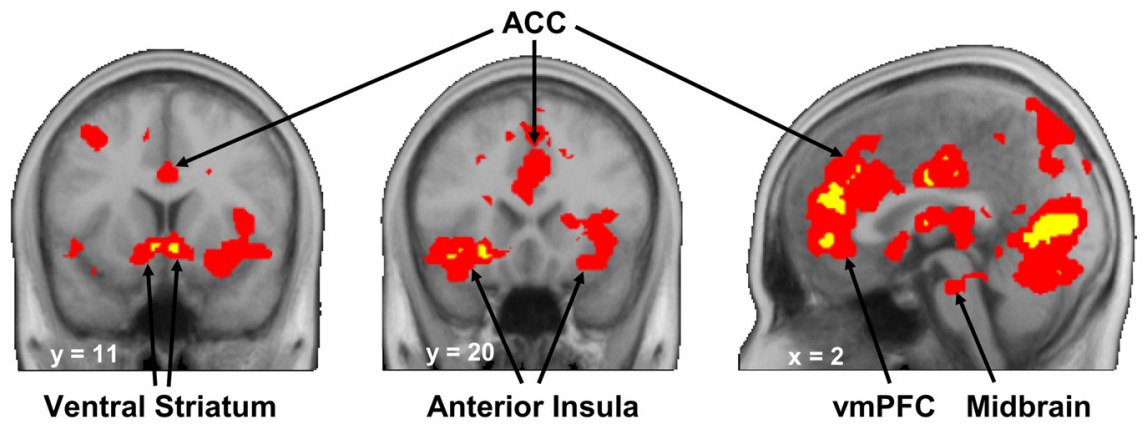

$p<0.01$ FDR $\square p<0.05$ FDR

Figure 5. Brain regions reflecting hedonic ratings regardless of reward type. Activations show the brain areas in which activity positively correlates with both monetary and erotic ratings (intersection of $T$-maps thresholded at $p<0.01$ FDR whole-brain corrected shown in yellow or thresholded at $p<0.05 \mathrm{FDR}$ whole-brain corrected shown in red). The regions displayed in red were used as an inclusive mask in the analysis of Figure 6 . Activations are overlaid on an average anatomical scan of all subjects.
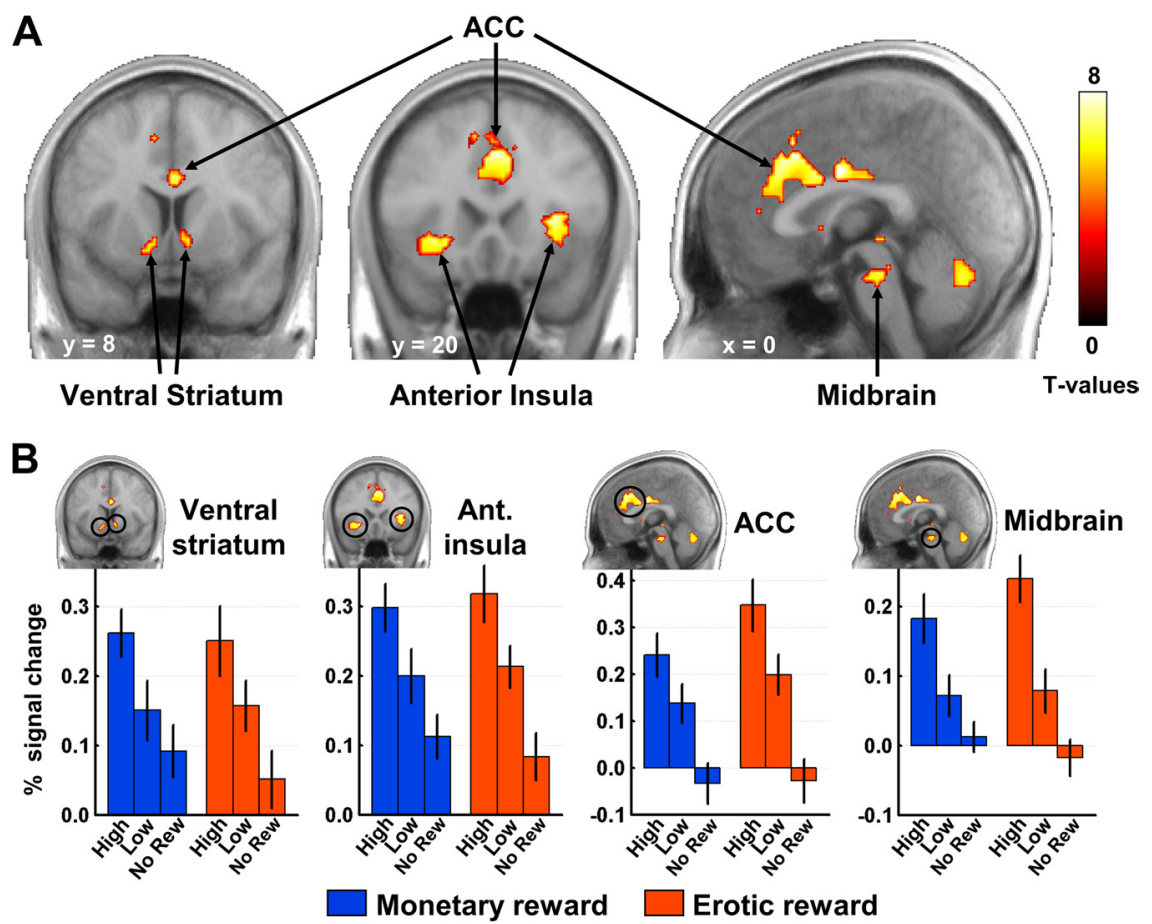

Figure 6. Common reward brain regions. $A, T$-map showing the brain regions encoding experienced reward value for both monetary and erotic reward outcomes. Activations are overlaid on an average anatomical scan of all subjects ( $p<0.05$ FWE whole-brain corrected). $\boldsymbol{B}$, Percent signal change is plotted in the circled ROls for monetary and erotic rewards according to the following conditions: high intensity, low intensity, and no reward. Note that these plots are not independent of the whole-brain analysis and are only shown as an illustration for easier visual comparison with Figure 4. Error bars indicate SEM. The signal is averaged across the right and left hemispheres in each brain region (similar patterns of activity were observed in each hemisphere).

$39], T=8.42)$, and the anterior insula $([-27,21,-6], T=7.48$; $[33,24,3], T=8.14$ ) (Fig. $6 A$ ). Other foci are reported in supplemental Table 3 (available at www.jneurosci.org as supplemental material).

The relative coding of reward intensity in these regions was further illustrated using the same ROI approach as with rewardspecific regions (Fig. 6B). Note, however, that this representation is not independent of the previous whole-brain analysis, because reward intensity is highly correlated with the hedonic ratings that have served in the identification of the "common network." Consequently, the resulting bar graphs are purely illustrative of the conclusion drawn from the $T$-map, which is that activity in the 


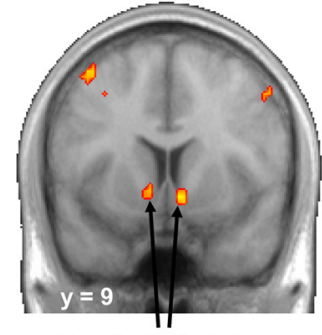

Ventral Striatum

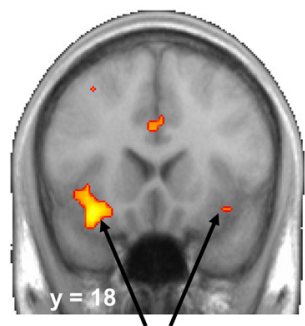

Anterior Insula

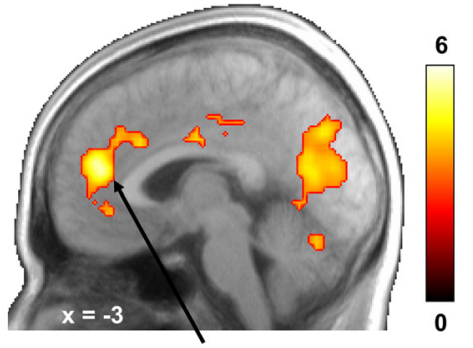

Rostral ACC

Figure 7. Brain regions reflecting prediction errors regardless of reward type. Activations result from a conjunction analysis showing the brain regions in which activity positively correlates with both monetary and erotic prediction errors $(p<0.001$ uncorrected). Activations are overlaid on an average anatomical scan of all subjects.

ventral striatum, midbrain, ACC, and anterior insula reflects experienced reward value regardless of reward type.

\section{Coding of reward prediction errors}

Reward-related brain regions are thought to mediate prediction error signals, coding the difference between expected outcomes and those effectively delivered (McClure et al., 2003; O'Doherty et al., 2003b; Schultz, 2006). These prediction error signals occur not only in conditioning procedures and can also be computed in non-learning situations, such as in the present study (Dreher et al., 2006; Yacubian et al., 2006). To determine whether such signals are supported by similar brain networks for monetary and erotic rewards, the fMRI data were fitted with parametric regressors modeling positive prediction errors related to reward outcomes. Our results revealed that monetary and erotic reward prediction errors were processed in a similar set of brain regions, essentially overlapping with the previously identified common network. Specifically, a conjunction analysis showed that activity in the ventral striatum $([-9,9,-6], T=3.98 ;[6,9,-9] T=$ $4.57)$, anterior insula $([-33,18,-18], T=4.80 ;[39,21,-15]$, $T=3.37)$, and rostral ACC $([-3,42,15], T=5.57)$ correlated positively with prediction errors regardless of reward type (Fig. 7) (supplemental Table 4, available at www.jneurosci.org as supplemental material). Moreover, the comparison of monetary and erotic reward prediction errors revealed almost no activity in reward-related areas (at $p<0.001$ uncorrected, only a tiny cluster appeared in the left pallidum for the contrast of erotic minus monetary prediction errors) and especially not in the brain regions labeled as "reward specific" (even at a liberal threshold of $p<0.05)$.

\section{Discussion}

As predicted, the OFC was found to be functionally organized along a postero-anterior axis with respect to reward type, with the anterior part responding exclusively to money and the posterior part responding exclusively to erotic stimuli. Additional eroticspecific activations were also found in the bilateral amygdala and medial OFC. Importantly, brain activity in these reward-specific regions only scaled with the hedonic value of the reward they specifically encoded. In parallel, our results support the idea of a core reward system processing experienced rewards regardless of their nature. In this common network, including the ventral striatum, ACC, anterior insula, and midbrain, functional activity correlated with hedonic value and prediction error for both monetary gains and erotic pictures. Together, our results reveal the existence of both reward-specific and nonspecific brain networks, challenging the view of a unique reward system for all reinforcers.
T-values

\section{Postero-anterior dissociation in the orbitofrontal cortex}

The segregated responses to monetary and erotic outcomes along a posteroanterior axis in the OFC suggest a functional division of experienced reward value representation according to an abstractness gradient. Paralleling this result in the domain of cognitive control, recent theories on the functional divisions of the human lateral prefrontal cortex proposed that it is organized hierarchically, whereby cognitive control involving temporally proximate and concrete action representations is supported by posterior lateral prefrontal regions, and cognitive control involving temporally extended and abstract representations is supported by more anterior lateral prefrontal regions such as the frontopolar cortex (Koechlin and Summerfield, 2007; Badre, 2008; Dreher et al., 2008a). Our study shows that a similar unifying principle of caudo-rostral hierarchical organization can be applied to the OFC. Notably, patients with lesions in the anterior OFC have been reported to be specifically impaired in making decisions entailing abstract, i.e., distant, consequences, and not in making decisions leading to concrete, i.e., immediate, consequences, further supporting a postero-anterior trend in the representation of abstractness in the OFC (Bechara and Damasio, 2005).

Although an anatomical gradient in the postero-anterior axis of the OFC had been suggested based on cytoarchitectonic data (Ongür and Price, 2000; Wise, 2008), its functional relevance for reward processing had never been tested empirically. Our data bring strong empirical support to this hypothesis. Although a generalization to all primary and secondary rewards cannot be ascertained in a single fMRI study, it is consistent with the pattern of activation observed in the recent literature for other rewards (Kringelbach and Rolls, 2004). In particular, the medial and posterior lateral OFC, responding to erotic pictures in the current and previous studies (Ponseti et al., 2006), were shown to respond to other primary rewards, such as attractive faces (O'Doherty et al., 2003a), pleasant odors (Gottfried et al., 2006), and pleasant taste (Small et al., 2001). Conversely, other studies manipulating monetary (Reuter et al., 2005; Vollm et al., 2007) or social (Izuma et al., 2008) rewards have reported a similar anterior OFC region as the one we found.

One intrinsic property of erotic pictures is that they are the reward, whereas monetary rewards delivered in the scanner are a representation of what the participant will receive at the end of the experiment. Thus, it could be argued that the OFC dissociation relates to the immediate rewarding effect of erotic pictures compared with the delayed rewarding effect of monetary gains. This is unlikely to be the case, because (1) our pattern of OFC activation was not observed for immediate versus delayed rewards in intertemporal choice studies (Kable and Glimcher, 2007; Prevost et al., 2010) and (2) the same OFC functional dissociation emerged when monetary and erotic rewards were expected but not effectively delivered. This finding also suggests that the segregated representation of reward types in the OFC was not merely attributable to visual or intrinsic differences between monetary and erotic outcomes, such as saliency or arousal. 
Electrophysiological recordings in monkeys indicate that OFC neurons encode the economic value assigned to different rewarding juices when choosing between them (PadoaSchioppa and Assad, 2008). Some OFC neurons were also found to encode taste responses reflecting the identity of a chosen juice. However, no neuronal recording study has yet investigated whether primary and secondary rewards (e.g., juice vs social dominance) are coded in distinct OFC subregions. Our finding of a clear OFC dissociation in humans supports a hierarchical organization along a continuum from the posterior to the anterior part of the OFC. Whether and how these distinct representations of value in the OFC are preserved across species remains an important open question. Our results also suggest that learning through secondary reinforcement may depend more on anterior OFC regions, whereas primary reinforcement may depend more on the posterior OFC. This hypothesis, which remains to be tested, may shed light on studies across a range of animal species indicating effects of OFC lesions on behavior maintained or acquired through secondary reinforcement (Murray et al., 2007).

In addition, our findings clarify how value signals in the OFC are integrated with those from other brain structures. Together with the posterior and medial OFC, we found that the amygdala responded exclusively to erotic pictures. Previous neuroimaging studies also reported that erotic pictures evoke amygdala response (Redouté et al., 2000; Karama et al., 2002; Hamann et al., 2004), whereas money, as a secondary reinforcer, often failed to do so in studies using experimental designs similar to the present paradigm (Knutson et al., 2001). This is consistent with the underlying anatomy, showing that the amygdala is more connected with the posterior and medial OFC than with the anterior OFC (Carmichael and Price, 1995). Moreover, the parametric modulation of the amygdala with erotic hedonic value is in accordance with its general role in emotional arousal for both appetitive and aversive stimuli. However, amygdala response to erotic stimuli is not solely determined by arousal, as suggested by a previous study reporting higher amygdala response in men than in women viewing sexual stimuli, despite similar arousal ratings in both groups (Hamann et al., 2004).

\section{Brain regions common to monetary and erotic rewards}

An important strength of our experimental design, compared with previous reward studies, is that it made it possible to directly test whether monetary and erotic reward outcomes are truly encoded within the same brain regions. The enhanced response to increasing hedonic value observed in the common network regardless of reward type suggests that this network processes experienced reward value in a general manner. This is consistent with its reported implication (in separate studies) in the hedonic processing of rewards ranging from primary reinforcers, such as sexual stimuli (Redouté et al., 2000), attractive faces (Bray and O'Doherty, 2007; Smith et al., 2010), and pleasant taste (Small et al., 2001), to secondary rewards, such as money or social approval (Izuma et al., 2008). Our common network activity is also compatible with an interpretation in terms of general arousal or saliency (Zink et al., 2004).

To efficiently compare the goal values of different rewards during decision making, it has been proposed that the brain may convert them into a common neural currency (Montague and Berns, 2002). A wealth of electrophysiological and fMRI studies has since confirmed this hypothesis, emphasizing in particular the role of the ventral striatum and vmPFC (Kable and Glimcher,
2007; Knutson et al., 2007; Chib et al., 2009). The present results suggest that a similar computation might be performed in the same brain network at the time of reward consumption. Indeed, along with the ventral striatum, activity in the vmPFC also reflected the hedonic experience of the participants regardless of reward type (Fig. 5). Such a mechanism encoding heterogeneous outcome values on a common scale might be helpful for efficient comparison of these values during subsequent value-based decision making.

Finally, we found prediction-error-related activity in the ventral striatum, anterior insula, and ACC for both monetary and erotic stimuli. This finding demonstrates that prediction errors are computed in a network independent of reward type and generalizes this concept to the domain of erotic stimuli, paralleling previous work performed with monetary gains (Yacubian et al., 2006), pleasant taste (O'Doherty et al., 2003b), and attractive faces (Bray and O'Doherty, 2007). Thus, prediction errors may be a primitive neural signal computed in midbrain dopaminergic neurons regardless of reward type and primarily delivered to the common reward network that may be responsible for making predictions. However, it is important to emphasize that, because of the inherent correlation existing between prediction error and hedonic value, it is difficult to disentangle these computations at the brain level (Behrens et al., 2008; Hare et al., 2008). Consequently, although the response pattern observed in our common network is consistent with both interpretations, we cannot ascribe with certainty one role or the other to these regions. Concerning the ventral striatum, however, a recent study aiming to dissociate reward value from prediction error found that activity in this region best correlated with the latter (Hare et al., 2008). Although this study focused on the computation of goal values in the context of decision making, and therefore cannot be directly compared with the present one, it brings evidence favoring the prediction error hypothesis in the ventral striatum. Note that midbrain activity was observed in our common network, but not in the prediction error analysis, whereas single-neuron recordings classically report prediction error signals in midbrain dopaminergic structures (Schultz, 2006). Although further work is needed to gain a better understanding of the relationship between dopaminergic neuron firing and the BOLD signal observed in reward paradigms, recent findings combining fMRI with FDOPA positron emission tomography measures of midbrain dopamine synthesis (Dreher et al., 2008b) and analyses of variations in genes involved in dopamine transmission established a link between higher prefronto-striatal BOLD signal and dopamine synaptic availability during reward processing (Dreher et al., 2009).

\section{Conclusion}

Our results provide plausible functional mechanisms explaining the existence of two separate reward networks in the brain. The nature of their interactions remains to be determined, but one possibility is that outcome value signals computed in the rewardspecific OFC regions would be sent to the common network for additional integration and comparison processes. From an evolutionary perspective, the distinct cytoarchitectonic properties of the anterior and posterior parts of the OFC suggest that the ability to process primary rewards may occur phylogenetically and ontogenetically earlier than the ability to process secondary rewards, which represent more evolved adaptive behavior. Our findings also have important clinical implications for a range of neuropsychopathological disorders characterized by major deficits in mo- 
tivation and behavioral control, such as pathological gambling or hypersexuality. The dissociable representation of various rewards along a postero-anterior axis in the OFC may shed light on this important question.

\section{References}

Abler B, Walter H, Erk S, Kammerer H, Spitzer M (2006) Prediction error as a linear function of reward probability is coded in human nucleus accumbens. Neuroimage 31:790-795.

Badre D (2008) Cognitive control, hierarchy, and the rostro-caudal organization of the frontal lobes. Trends Cogn Sci 12:193-200.

Bechara A, Damasio A (2005) The somatic marker hypothesis: A neural theory of economic decision. Games Econ Behav 52:336-372.

Beck AT, Beck RW (1972) Screening depressed patients in family practice. A rapid technic. Postgrad Med 52:81-85.

Behrens TE, Hunt LT, Woolrich MW, Rushworth MF (2008) Associative learning of social value. Nature 456:245-249.

Bray S, O'Doherty J (2007) Neural coding of reward-prediction error signals during classical conditioning with attractive faces. J Neurophysiol 97:3036-3045.

Caldú X, Dreher JC (2007) Hormonal and genetic influences on processing reward and social information. Ann NY Acad Sci 1118:43-73.

Carmichael ST, Price JL (1995) Limbic connections of the orbital and medial prefrontal cortex in macaque monkeys. J Comp Neurol 363:615-641.

Chib VS, Rangel A, Shimojo S, O’Doherty JP (2009) Evidence for a common representation of decision values for dissimilar goods in human ventromedial prefrontal cortex. J Neurosci 29:12315-12320.

Deaner RO, Khera AV, Platt ML (2005) Monkeys pay per view: adaptive valuation of social images by rhesus macaques. Curr Biol 15:543-548.

Dreher JC (2009) Decomposing brain signals involved in value-based decision making. In: Handbook of reward and decision making (Dreher JC, Tremblay L, eds), pp 137-164. New York: Academic/Elsevier.

Dreher JC, Kohn P, Berman KF (2006) Neural coding of distinct statistical properties of reward information in humans. Cereb Cortex 16:561-573.

Dreher JC, Schmidt PJ, Kohn P, Furman D, Rubinow D, Berman KF (2007) Menstrual cycle phase modulates reward-related neural function in women. Proc Natl Acad Sci U S A 104:2465-2470.

Dreher JC, Koechlin E, Tierney M, Grafman J (2008a) Damage to the fronto-polar cortex is associated with impaired multitasking. PLoS One 3:e3227.

Dreher JC, Meyer-Lindenberg A, Kohn P, Berman KF (2008b) Age-related changes in midbrain dopaminergic regulation of the human reward system. Proc Natl Acad Sci U S A 105:15106-15111.

Dreher JC, Kohn P, Kolachana B, Weinberger DR, Berner KF (2009) Variation in dopamine genes influences responsivity of the human reward system. Proc Natl Acad Sci U S A 106:617-622.

FitzGerald TH, Seymour B, Dolan RJ (2009) The role of human orbitofrontal cortex in value comparison for incommensurable objects. J Neurosci 29:8388-8395.

Gomes CM, Boesch C (2009) Wild chimpanzees exchange meat for sex on a long-term basis. PLoS One 4:e5116.

Gottfried JA, Small DM, Zald DH (2006) The chemical senses. The orbitofrontal cortex, pp 125-171. Oxford: Oxford UP.

Hamann S, Herman RA, Nolan CL, Wallen K (2004) Men and women differ in amygdala response to visual sexual stimuli. Nat Neurosci 7:411-416.

Hammers A, Allom R, Koepp MJ, Free SL, Myers R, Lemieux L, Mitchell TN, Brooks DJ, Duncan JS (2003) Three-dimensional maximum probability atlas of the human brain, with particular reference to the temporal lobe. Hum Brain Mapp 19:224-247.

Hare TA, O’Doherty J, Camerer CF, Schultz W, Rangel A (2008) Dissociating the role of the orbitofrontal cortex and the striatum in the computation of goal values and prediction errors. J Neurosci 28:5623-5630.

Hayden BY, Parikh PC, Deaner RO, Platt ML (2007) Economic principles motivating social attention in humans. Proc Biol Sci 274:1751-1756.

Henson R (2006) Forward inference using functional neuroimaging: dissociations versus associations. Trends Cogn Sci 10:64-69.

Hoon E, Chambless D (1998) Sexual arousability inventory and sexual arousability inventory-expanded. In: Handbook of SexualityRelated Measures, pp 71-74. Washington, DC: American Psychiatric Association.
Hunt LT (2008) Distinctive roles for the ventral striatum and ventral prefrontal cortex during decision-making. J Neurosci 28:8658-8659.

Izuma K, Saito DN, Sadato N (2008) Processing of social and monetary rewards in the human striatum. Neuron 58:284-294.

Kable JW, Glimcher PW (2007) The neural correlates of subjective value during intertemporal choice. Nat Neurosci 10:1625-1633.

Karama S, Lecours AR, Leroux JM, Bourgouin P, Beaudoin G, Joubert S, Beauregard M (2002) Areas of brain activation in males and females during viewing of erotic film excerpts. Hum Brain Mapp 16:1-13.

Knutson B, Fong GW, Adams CM, Varner JL, Hommer D (2001) Dissociation of reward anticipation and outcome with event-related fMRI. Neuroreport 12:3683-3687.

Knutson B, Taylor J, Kaufman M, Peterson R, Glover G (2005) Distributed neural representation of expected value. J Neurosci 25:4806-4812.

Knutson B, Rick S, Wimmer GE, Prelec D, Loewenstein G (2007) Neural predictors of purchases. Neuron 53:147-156.

Koechlin E, Summerfield C (2007) An information theoretical approach to prefrontal executive function. Trends Cogn Sci 11:229-235.

Kriegeskorte N, Simmons WK, Bellgowan PS, Baker CI (2009) Circular analysis in systems neuroscience: the dangers of double dipping. Nat Neurosci 12:535-540

Kringelbach ML, Rolls ET (2004) The functional neuroanatomy of the human orbitofrontal cortex: evidence from neuroimaging and neuropsychology. Prog Neurobiol 72:341-372.

McClure SM, Berns GS, Montague PR (2003) Temporal prediction errors in a passive learning task activate human striatum. Neuron 38:339-346.

McClure SM, Ericson KM, Laibson DI, Loewenstein G, Cohen JD (2007) Time discounting for primary rewards. J Neurosci 27:5796-5804.

Montague PR, Berns GS (2002) Neural economics and the biological substrates of valuation. Neuron 36:265-284

Murray EA, O'Doherty JP, Schoenbaum G (2007) What we know and do not know about the functions of the orbitofrontal cortex after 20 years of cross-species studies. J Neurosci 27:8166-8169.

Nichols T, Brett M, Andersson J, Wager T, Poline JB (2005) Valid conjunction inference with the minimum statistic. Neuroimage 25:653-660.

O’Doherty J, Winston J, Critchley H, Perrett D, Burt DM, Dolan RJ (2003a) Beauty in a smile: the role of medial orbitofrontal cortex in facial attractiveness. Neuropsychologia 41:147-155.

O’Doherty JP, Dayan P, Friston K, Critchley H, Dolan RJ (2003b) Temporal difference models and reward-related learning in the human brain. Neuron 38:329-337.

Ongür D, Price JL (2000) The organization of networks within the orbital and medial prefrontal cortex of rats, monkeys and humans. Cereb Cortex 10:206-219.

Padoa-Schioppa C, Assad JA (2008) The representation of economic value in the orbitofrontal cortex is invariant for changes of menu. Nat Neurosci 11:95-102.

Peters J, Büchel C (2010) Neural representations of subjective reward value. Behav Brain Res 213:135-141.

Plassmann H, O’Doherty J, Rangel A (2007) Orbitofrontal cortex encodes willingness to pay in everyday economic transactions. J Neurosci 27:9984-9988.

Ponseti J, Bosinski HA, Wolff S, Peller M, Jansen O, Mehdorn HM, Büchel C, Siebner HR (2006) A functional endophenotype for sexual orientation in humans. Neuroimage 33:825-833.

Prevost C, Pessiglione M, Météreau E, Clery-Melin ML, Dreher JC (2010) Distinct valuation subsystems in the human brain for effort and delay. J Neurosci, in press.

Rangel A, Camerer C, Montague PR (2008) A framework for studying the neurobiology of value-based decision making. Nat Rev Neurosci 9:545-556.

Redouté J, Stoléru S, Grégoire MC, Costes N, Cinotti L, Lavenne F, Le Bars D, Forest MG, Pujol JF (2000) Brain processing of visual sexual stimuli in human males. Hum Brain Mapp 11:162-177.

Reuter J, Raedler T, Rose M, Hand I, Gläscher J, Büchel C (2005) Pathological gambling is linked to reduced activation of the mesolimbic reward system. Nat Neurosci 8:147-148.

Reynolds CF 3rd, Frank E, Thase ME, Houck PR, Jennings JR, Howell JR, Lilienfeld SO, Kupfer DJ (1988) Assessment of sexual function in depressed, impotent, and healthy men: factor analysis of a brief sexual function questionnaire for men. Psychiatry Res 24:231-250.

Rhodes G (2006) The evolutionary psychology of facial beauty. Annu Rev Psychol 57:199-226. 
Schultz W (2006) Behavioral theories and the neurophysiology of reward. Annu Rev Psychol 57:87-115.

Small DM, Zatorre RJ, Dagher A, Evans AC, Jones-Gotman M (2001) Changes in brain activity related to eating chocolate: from pleasure to aversion. Brain 124:1720-1733.

Smith DV, Hayden BY, Truong TK, Song AW, Platt ML, Huettel SA (2010) Distinct value signals in anterior and posterior ventromedial prefrontal cortex. J Neurosci 30:2490-2495.

Sugrue LP, Corrado GS, Newsome WT (2005) Choosing the greater of two goods: neural currencies for valuation and decision making. Nat Rev Neurosci 6:363-375.

Thornhill R, Gangestad SW (1999) Facial attractiveness. Trends Cogn Sci 3:452-460.
Tremblay L, Schultz W (1999) Relative reward preference in primate orbitofrontal cortex. Nature 398:704-708.

Völlm B, Richardson P, McKie S, Elliott R, Dolan M, Deakin B (2007) Neuronal correlates of reward and loss in Cluster B personality disorders: a functional magnetic resonance imaging study. Psychiatry Res 156:151-167.

Wise SP (2008) Forward frontal fields: phylogeny and fundamental function. Trends Neurosci 31:599-608.

Yacubian J, Gläscher J, Schroeder K, Sommer T, Braus DF, Büchel C (2006) Dissociable systems for gain- and loss-related value predictions and errors of prediction in the human brain. J Neurosci 26:9530-9537.

Zink CF, Pagnoni G, Martin-Skurski ME, Chappelow JC, Berns GS (2004) Human striatal responses to monetary reward depend on saliency. Neuron 42:509-517. 